\title{
Cortical Oscillatory Activity Is Critical for Working Memory as Revealed by Deficits in Early-Onset Schizophrenia
}

\author{
Corinna Haenschel, ${ }^{1,2,3}$ Robert A. Bittner, ${ }^{1,2,4}$ James Waltz, ${ }^{5}$ Fabian Haertling, ${ }^{6}$ Michael Wibral, ${ }^{4}$ Wolf Singer, ${ }^{2}$ \\ David E. J. Linden, ${ }^{3}$ and Eugenio Rodriguez ${ }^{2,7}$ \\ ${ }^{1}$ Laboratory for Neurophysiology and Neuroimaging, Department of Psychiatry, Johann Wolfgang Goethe University, 60590 Frankfurt, Germany, ${ }^{2}$ Max \\ Planck Institute for Brain Research, 60528 Frankfurt, Germany, ${ }^{3}$ School of Psychology, Bangor University, Gwynedd LL57 2AS, United Kingdom, ${ }^{4}$ Brain \\ Imaging Center, 60528 Frankfurt, Germany, ${ }^{5}$ University of Maryland School of Medicine, Baltimore, Maryland 21228, ${ }^{6}$ Department of Child and Adolescent \\ Psychiatry, Johann Wolfgang Goethe University, 60528 Frankfurt, Germany, and PPontificia Universidad Católica de Chile, Escuela de Psicología, 7820436 \\ Macul, Santiago, Chile
}

Impairments in working memory (WM) are a core cognitive deficit in schizophrenia. Neurophysiological models suggest that deficits during WM maintenance in schizophrenia may be explained by abnormalities in the GABAergic system, which will lead to deficits in high-frequency oscillations. However, it is not yet clear which of the three WM phases (encoding, maintenance, retrieval) are affected by dysfunctional oscillatory activity. We investigated the relationship between impairments in oscillatory activity in a broad frequency range $(3-100 \mathrm{~Hz})$ and WM load in the different phases of WM in 14 patients with early-onset schizophrenia and 14 matched control participants using a delayed matching to sample paradigm.

During encoding, successful memorization was predicted by evoked theta, alpha, and beta oscillatory activity in controls. Patients showed severe reductions in the evoked activity in these frequency bands. During early WM maintenance, patients showed a comparable WM load-dependent increase in induced alpha and gamma activity to controls. In contrast, during the later maintenance phase, patients showed a shift in the peak of induced gamma activity to the lower WM load conditions. Finally, induced theta and gamma activity were reduced in patients during retrieval.

Our findings suggest that the WM deficit in schizophrenia is associated with impaired oscillatory activity during all phases of the task and that the cortical storage system reaches its capacity limit at lower loads. Inability to maintain oscillatory activity in specific frequency bands could thus result in the information overload that may underlie both cognitive deficits and psychopathological symptoms of schizophrenia.

\section{Introduction}

Working memory (WM) deficits are a cardinal neuropsychological feature of schizophrenia (Tallon-Baudry et al., 1998; Goldman-Rakic, 2001; Silver et al., 2003; Gooding and Tallent, 2004). According to a previous meta-analysis, encoding and the early part of the maintenance seem to be the most affected subprocesses of WM (Lee and Park, 2005). Several behavioral studies have shown impaired performance in patients with schizophrenia linked to dysfunctional WM encoding (Javitt et al., 1997; Lencz et al., 2003; Lee and Park, 2005). Furthermore, we provided evidence that the event-related potential (ERP) component P100 is of relevance for successful WM encoding in controls and is

Received March 24, 2009; revised June 3, 2009; accepted June 15, 2009.

This work was supported by the Bundesministeriums für Bildung und Forschung (Federal Ministry of Education and Research). E.R. was partially supported by Fondo Nacional de Desarrollo Científico y Tecnológico Grant 1070846 and a joint collaboration grant with C.H. from La Comisión Nacional de Investigación Científica y Tecnológica/ Deutscher Akademischer Austausch Dienst. C.H. was supported by the Wales Institute of Cognitive Neuroscience, funded by the Welsh Assembly Government. We thank Petra Janson from the Max Planck Institute for Brain Research for help with the artwork and Niko Kriegeskorte for generating the stimuli.

Correspondence should be addressed to Corinna Haenschel, School of Psychology, Adeilad Brigantia, Bangor University, Gwynedd LL57 2AS, UK. E-mail: c.haenschel@bangor.ac.uk.

D0I:10.1523/JNEUROSCI.1428-09.2009

Copyright $\odot 2009$ Society for Neuroscience $\quad 0270-6474 / 09 / 299481-09 \$ 15.00 / 0$ reduced in adolescent patients with schizophrenia (Haenschel et al., 2007b). WM maintenance deficits in schizophrenia have also been observed both behaviorally (Tek et al., 2002; Badcock et al., 2008) and using functional magnetic resonance imaging (fMRI), especially in the dorsolateral prefrontal cortex (DLPFC) (Cannon et al., 2005; Lee et al., 2008) using spatial delayed response tasks.

These neurophysiological and functional neuroimaging studies of visual WM have demonstrated that distinct cortical areas have particular importance for specific stages of WM. However, the underlying neurophysiological processes that link activity associated with WM within and across cortical areas still remain poorly understood. Synchronized high-frequency oscillatory activity may provide a flexible link to integrate neural activity within and between cortical areas, for a wide range of cognitive functions (Singer, 1999), including WM (Tallon-Baudry et al., 1998).

Such oscillatory activity is generated by networks of interconnected GABAergic interneurons (Whittington et al., 1995). Postmortem work in schizophrenia has shown reduced GABAergic and glutamatergic neurotransmission in certain DLPFC microcircuits (Lewis, 2000; Lewis and Moghaddam, 2006) and recently throughout the neocortex (Hashimoto et al., 2008). This should 
impair high-frequency oscillatory activity and could result in impaired perception and cognition. Such a direct link between alterations in markers of GABAergic and glutamatergic neurotransmission and, in particular, gamma oscillatory activity has been found in animal models of schizophrenia (Cunningham et al., 2006; Roopun et al., 2008), demonstrating a deficit in gamma rhythmogenesis. Furthermore, there is evidence of abnormal gamma band activity $(30-100 \mathrm{~Hz})$ in human EEG during both perceptual tasks (Kwon et al., 1999; Spencer et al., 2003; Light et al., 2006) and WM maintenance (Cho et al., 2006; Basar-Eroglu et al., 2007) in patients with schizophrenia. In addition, abnormal oscillatory activity during WM has also been reported in the theta $(3-7 \mathrm{~Hz})$ (Schmiedt et al., 2005) and alpha (8-12 Hz) (Bachman et al., 2008) frequency bands (Haenschel et al., 2007a).

These WM studies used either a combination of cognitive control and N-back tasks (which makes it impossible to isolate the activity related to distinct WM subprocesses) or investigated only the delay period. Hence, the effects of oscillatory activity across all stages of a pure WM task and across longer delay periods still need to be investigated. Furthermore, the differences in frequency bands reported across the studies highlight the need to examine a wider range of frequencies in the same task.

The aim of the current study was to clarify which of the three WM phases (encoding, maintenance, retrieval) exhibit dysfunctional oscillatory activity in patients with schizophrenia and how these neural deficits would relate to the behavioral impairment. Finally, we examined whether oscillatory activity is reduced over a longer delay interval than has previously been investigated.

\section{Materials and Methods}

\section{Subjects}

Fourteen patients with early-onset schizophrenia [EOS; i.e., an onset of the disorder before the age of 18, diagnosed according to DSM-IV (Diagnostic and Statistical Manual of Mental Disorders, 4th Edition) criteria] [ 9 males, 5 females; mean age, 17.76 (SD, 1.44); 11 right handed, 3 left handed; age at onset, 16.44 (SD, 1.24); illness duration, 1.26 (SD, 0.8); premorbid intelligence quotient (IQ), 97 (SD, 14)] using the Mehrfachwahl-Wortschatz-Intelligenztest (Lehrl, 1995) and 14 matched control participants [7 males, 7 females; mean age, 17.37 (SD, 1.41); 10 right handed, 4 left handed; premorbid IQ, 95 (SD, 8)] underwent EEG recordings during a visual WM task. Current clinical symptoms of patients were assessed with the Positive and Negative Syndrome Scale (mean score, 43; SD, 9.31) (Kay et al., 1987). All patients were on medication at the time of testing, with 13 of 14 receiving atypical antipsychotic medication (Quetiapine, 10; Risperidone, 1; Clozapine, 1; Olanzapine, 1). The mean equivalent medication level as measured in chlorpromazine equivalents (Woods, 2003) was $163.02 \mathrm{mg} / \mathrm{d}$. One patient received selective serotonin reuptake inhibitors as well. All adult participants provided informed consent before the study. In addition, for participants under 18 years, parents assented to participation. All participants with a history of substance abuse 6 months preceding the study or an additional neuropsychiatric diagnosis in the case of patients or any neuropsychiatric diagnoses in the case of controls were excluded. Ethical approval was obtained from the ethics committee of the Medical School, Johann Wolfgang Goethe University (Frankfurt am Main, Germany).

\section{Stimuli and task}

A delayed discrimination task that probes load effects in visual WM (Linden et al., 2003) with 36 non-natural visual objects [blurred outlines of random tetris shapes (BORTS)] that were presented in the center of the computer monitor (visual angle, $1.34^{\circ}$ ) was implemented on a personal computer using the Experimental-Run-Time-System software (Fig. 1). BORTs have the advantage of being novel and difficult to verbalize. Two EEG sessions, each comprising three 10 min blocks, were held on consecutive days. Trials with different WM load levels were randomly distributed across sessions with a total of 50 trials obtained per WM load level. The ERP data have been reported previously (Haenschel et al., 2007b).

\section{Measurement and analysis}

A 64-channel electrode cap was fitted to the participants' head with the ground electrode at position AFZ and the reference electrode at position FCZ. An additional vertical electro-oculogram electrode was placed below the right eye. Electrode impedance was kept below $5 \mathrm{k} \Omega$. Recording, digitization, and preprocessing of the EEG data were performed with a BrainAmp amplifier and the BrainVision Recorder software (Brain Products). The EEG was recorded at a sampling rate of $500 \mathrm{~Hz}$ with a system bandpass between 0 and $100 \mathrm{~Hz}$.

EEG data analysis. EEG data were referenced to linked electrodes TP9TP10. This reference was chosen because it was unlikely to be involved in widespread synchronous activity (Rodriguez et al., 1999). Independent component analysis (ICA) was used to identify eyeblink artifacts by their distinct topography and to remove them from the data using the BrainVision Analyser software (Brain Products). Epochs were excluded automatically if the amplitudes exceeded a threshold of $\pm 100 \mu \mathrm{V}$.

Following the recent report of Yuval-Greenberg et al. (2008; Melloni et al., 2009), the possibility of a relationship between induced gamma band activity and microsaccades must be considered. We performed ICA decomposition and looked for components that (1) had the properties of microsaccades (irregular appearance over trials, broadband spectrum, frontal topography in average reference) and (2) accounted for the observed effects in the gamma band. We indeed found components with microsaccade-like properties (1), but these components exhibited power-time courses that differed from the observed effects in the gamma band. We concluded that induced gamma band activity in our data was not explained by microsaccades.

Encoding. To assess encoding, we only analyzed the last sample stimulus in each WM load condition (i.e., the only stimulus for WM load 1, the second stimulus for WM load 2, and the third for WM load 3). This ensured an equal number of stimuli for each condition and, more importantly, maximized the effect of prior processing in the higher WM load conditions. The baseline was -600 to $0 \mathrm{~ms}$ relative to trial onset, followed by the analysis up to $3 \mathrm{~s}$. For WM load 1, we analyzed the first 
$1000 \mathrm{~ms}$; for WM load 2, we analyzed the time period between 1000 and $2000 \mathrm{~ms}$ ( $1000 \mathrm{~ms}$ onset of stimulus 2); and for WM load 3, we analyzed the period between 2000 and $3000 \mathrm{~ms}$ (2000 ms onset of stimulus 3 ) after stimulus onset.

Maintenance. To assess maintenance, we defined two delay windows between 500 and $2500 \mathrm{~ms}$ and between 2500 and $4500 \mathrm{~ms}$ after stimulus offset. The baseline was -600 to $0 \mathrm{~ms}$ relative to the offset of stimuli 1-3 for WM loads $1-3$, respectively.

Retrieval. To assess retrieval-related activity, we set the baseline to -600 to $0 \mathrm{~ms}$ relative to the probe stimulus onset, and we analyzed the $1000 \mathrm{~ms}$ following the stimulus.

After the segmentation, all correct trials were entered into the timefrequency analysis using Matlab scripts developed in house, based on Lachaux et al. (1999). The digitized signals were analyzed by means of a windowed Fourier transform (window length, 150 points for the analysis of $10-100 \mathrm{~Hz}$ and 250 points for the analysis of $1-20 \mathrm{~Hz}$; step 14 points; window overlap, 90\%). Signal windows were zero padded to 512 points to obtain an interpolated frequency resolution of $\sim 1 \mathrm{~Hz}$ per frequency bin. For every time window and frequency bin, we computed amplitudes (for methods, see Uhlhaas et al., 2006).

In a second step, we computed the evoked and the induced oscillatory activity over two regions of interest (ROIs): 1) an anterior ROI: F1, FZ, F2, FC1, FCZ, FC2, C1, CZ, C2; and a posterior ROI: P3, PZ, P4, PO3, $\mathrm{POZ}, \mathrm{PO} 4, \mathrm{O} 1, \mathrm{Oz}, \mathrm{O} 2$. For the evoked activity, we first averaged across the trials before computing the time-frequency plots; for the induced oscillatory activity, we first computed the time-frequency distribution on single trials, subtracted the evoked part from the single trials, and finally averaged.

Statistical analysis. Behavioral data were analyzed with a two-way ANOVA with the dependent-variables reaction time and the estimated number of successfully encoded objects and the factors WM load and group. We computed the number of successfully encoded objects using Pashler's formula (Pashler, 1988; Cowan et al., 2005): $s=n^{*}(h-g) /(1-g)$, where $s$ is the number of stored items, $n$ is the number of items in the display ( 1 in memory load 1, 2 in memory load 2 , etc.), $h$ is the hit rate (correctly identified matches), and $g$ is the rate of false alarms (nonmatches incorrectly identified as matches).

The statistical analysis of EEG data was based on the assumption that encoding, early and late delay activity, and retrieval would relate to oscillatory activity and may help to elucidate the differences between controls and schizophrenic patients. We analyzed evoked and induced activity in the theta, alpha, beta, and gamma frequency bands. In contrast to most previous studies, which focused on a limited frequency range, we measured evoked and induced oscillatory activity for the full range between 3 and $100 \mathrm{~Hz}$. We will, however, only report significant latency and amplitude effects.

During stimulus encoding, we observed evoked beta activity (12-30 $\mathrm{Hz}$; analyzed between 30 and $350 \mathrm{~ms}$ after the onset of stimulus 1, 1030 and $1350 \mathrm{~ms}$ for stimulus 2, and 2030 and $2350 \mathrm{~ms}$ for stimulus 3 ) and evoked alpha $(8-12 \mathrm{~Hz})$ and theta $(3-7 \mathrm{~Hz})$ activity (both analyzed between 30 and $500 \mathrm{~ms}$ after the onset of stimulus 1, 1030 and $1500 \mathrm{~ms}$ for stimulus 2, and 2030 and $2500 \mathrm{~ms}$ for stimulus 3). During the first $5 \mathrm{~s}$ of the delay, we observed induced alpha activity $(11-13 \mathrm{~Hz})$ and induced gamma activity $(55-100 \mathrm{~Hz}$ ) (both analyzed in an early delay interval between 500 and $2500 \mathrm{~ms}$ and in a later delay interval between 2500 and $4500 \mathrm{~ms}$ after stimulus offset). During retrieval, we observed evoked theta activity (analyzed between 30 and $500 \mathrm{~ms}$ ), followed by induced theta (analyzed between 200 and $600 \mathrm{~ms}$ ) and gamma (analyzed between 200 and $800 \mathrm{~ms}$ ) oscillatory activity.

Repeated-measures multivariate ANOVA was used to test the effects within ROI (nine anterior and nine posterior electrodes) and WM load and between participants (participant group) on all dependent mean amplitude and peak latency measures. We then analyzed which of the subprocesses and which frequency components would best predict the estimated number of successfully encoded objects. This was addressed using multiple hierarchical regression with the estimated number of successfully encoded objects serving as the dependent variable and the evoked and induced frequency components for encoding, maintenance, and retrieval in separate blocks serving as predictor

\section{Reaction Time}

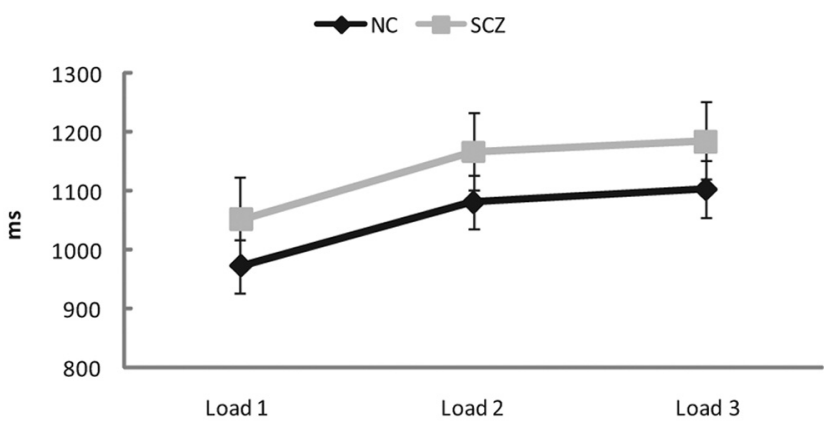

Number of Encoded Items

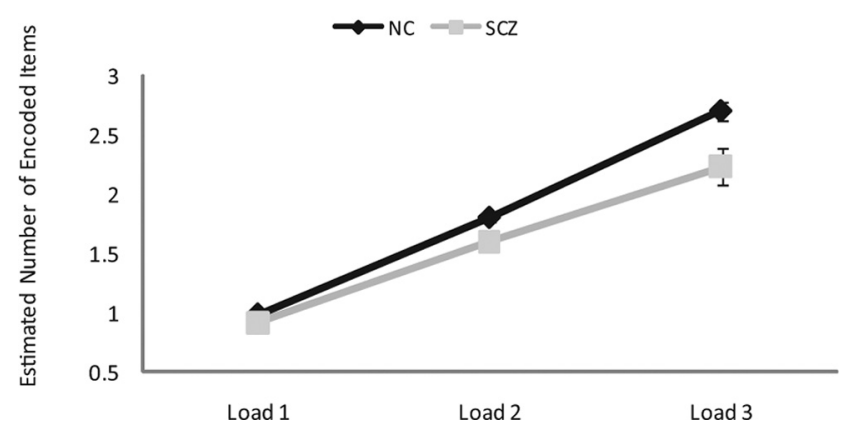

Figure 2. Behavioral data (reaction time and number of successfully encoded items). The mean reaction time (top) and number of successfully encoded items (bottom) in response to a WM load of one, two, or three in controls (black line) and patients (gray line) are shown. Error bars represent SE. NC, Normal controls; SCZ, patients with early-onset schizophrenia.

variables. The regression analysis was performed for each group separately. Furthermore, we correlated the chlorpromazine equivalents with the dependent measures.

\section{Results}

\section{Behavior}

Figure 2 shows the average reaction times and the number of successfully encoded objects for controls and patients. Reaction time increased with WM load for both groups $\left(F_{(2,25)}=132.65\right.$; $p<0.001)$. The control group demonstrated a greater increase with WM load than patients (load $\times$ group: $F_{(2,25)}=3.53 ; p=$ $0.041)$. The linear contrasts confirmed the monotonic increase in both groups with WM load $\left(F_{(1,26)}=196.33 ; p<0.001\right)$.

The number of successfully encoded items was significantly lower for patients compared with controls $\left(F_{(1,26)}=8.98 ; p=\right.$ $0.006)$. Both groups showed a linear increase in retained items with the increasing WM load $\left(F_{(1,26)}=242.89 ; p<0.001\right)$, but the increase was stronger in controls compared with patients. This was statistically supported by a significant WM load $\times$ group interaction $\left(F_{(2,25)}=4.4 ; p=0.027\right)$.

There was no correlation between chlorpromazine equivalents and reaction time or the number of successfully encoded objects.

\section{Time-frequency analysis of EEG signal energy}

Encoding

The grand mean evoked beta, alpha, and theta band activity at posterior electrodes in response to WM loads 1-3 during encoding in controls and patients is illustrated in Figure 3.

The sample stimuli (i.e., stimulus 1 of WM load 1, stimulus 2 of WM load 2, and stimulus 3 of WM load 3) elicited evoked $\beta$ band activity $(12-30 \mathrm{~Hz})$ between 30 and $350 \mathrm{~ms}$ and evoked 

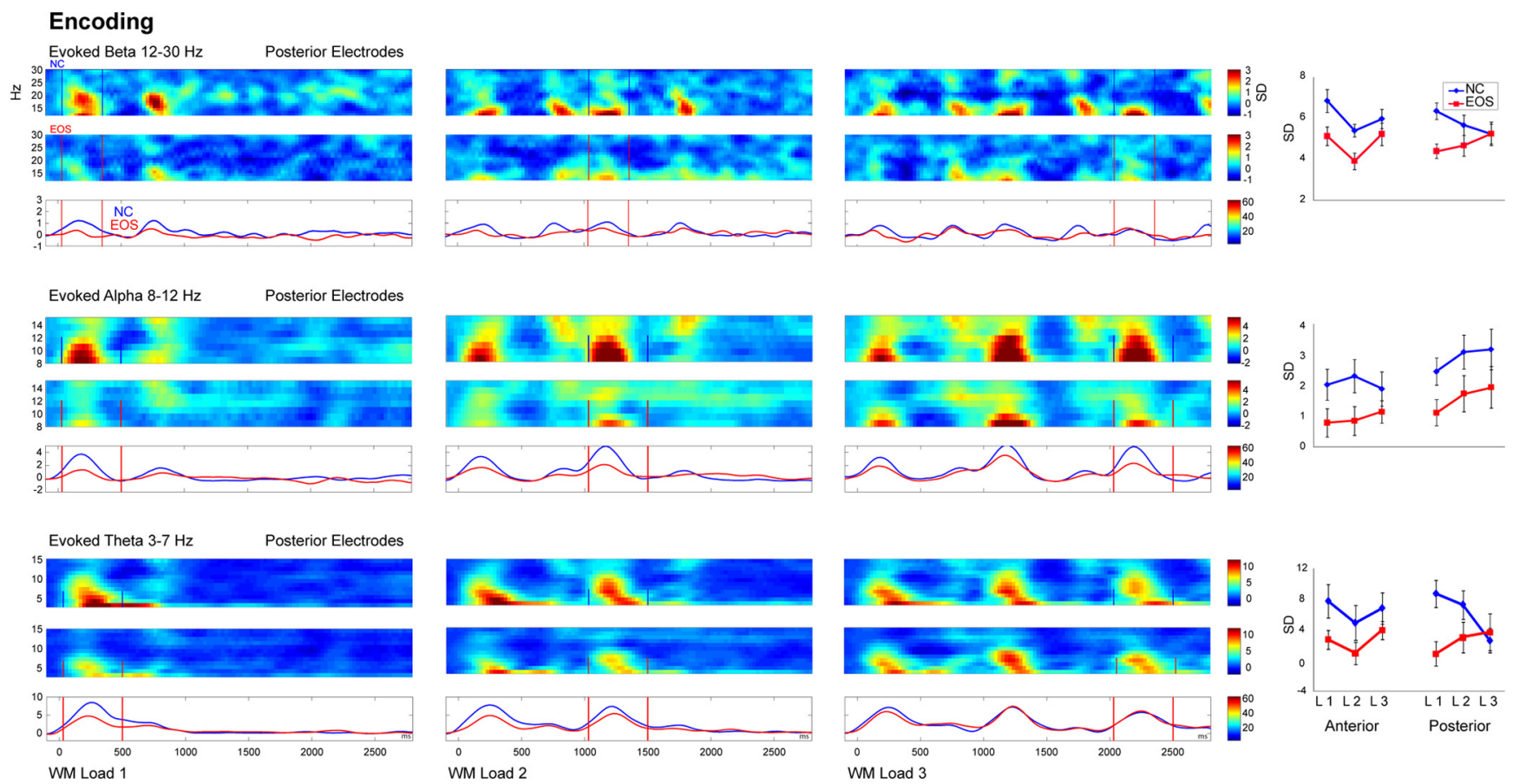

Figure 3. Evoked oscillatory activity during WM encoding. Evoked beta (top), alpha (middle), and theta (bottom) activity after WM load 1 (left), WM load 2 (center), and WM load 3 (right) is shown for the encoding period of $3 \mathrm{~s}$ at posterior electrodes for controls ( $\mathrm{NC}$, blue, top row) and patients with EOS (red, middle row) and the group comparison in the frequency range and time window of interest (bottom row). The frequency range and the window of interest (the first sample stimulus for WM load 1, the second stimulus for WM load 2, and the third stimulus for WM load 3 are denoted in bars) are shown. On the far right, mean amplitudes in response to WM load 1, 2, or 3 are shown for the anterior and posterior ROI for patients with EOS (red) and controls (NC, blue). Error bars represent SE.

theta $(3-7 \mathrm{~Hz})$ and alpha $(8-12 \mathrm{~Hz})$ band activity between 30 and $500 \mathrm{~ms}$ in both groups (supplemental Table 1, available at www. jneurosci.org as supplemental material). Evoked alpha and theta band activity peaked earlier at posterior compared with anterior electrodes (alpha: $F_{(1,26)}=7.97, p=0.009$; theta: $F_{(1,26)}=8.63$, $p=0.007)$. Evoked beta band activity did not show any difference in latency.

Patients showed reduced evoked theta $\left(F_{(1,26)}=8.87 ; p=0.006\right)$, alpha $\left(F_{(1,26)}=5.34 ; p=0.029\right)$, and in-trend beta $\left(F_{(1,26)}=3.93\right.$; $p=0.058)$ band amplitudes compared with controls.

Furthermore, controls but not patients exhibited a decrease in evoked beta amplitude at posterior electrodes with increasing WM load $\left(\mathrm{WM}\right.$ load $\times$ ROI $\times$ group: $\left.F_{(2,25)}=4.9 ; p=0.036\right)$. Evoked theta band showed a trend for an interaction between WM load and ROI (WM load $\times$ ROI: $\left.F_{(2,25)}=3.05 ; p=0.064\right)$. Post hoc tests indicated that evoked theta band activity decreased linearly in amplitude with increasing WM load in controls at posterior electrodes $\left(\mathrm{WM}\right.$ load $\times$ group: $\left.F_{(2,25)}=6.3 ; p=0.019\right)$ similar to evoked beta band activity. In addition, evoked theta band activity exhibited a quadratic decrease at anterior electrodes for both groups $\left(F_{(2,25)}=13.51 ; p=0.001\right)$.

Given that evoked oscillatory activity in the delta to beta range has been associated with the early event-related P1-N1 components in healthy subjects (Klimesch et al., 2004; Gruber et al., 2005), we hypothesized that the deficits in evoked oscillatory activity in the theta, alpha, and beta range reported here may be related to the $\mathrm{P} 1$ deficit in EOS patients, as reported previously (Haenschel et al., 2007b). To test this hypothesis, we performed post hoc correlation between the $\mathrm{P} 1$ peak amplitude and the mean evoked theta, alpha, and beta amplitudes. The results showed a significant positive correlation between $\mathrm{P} 1$ and posterior theta activity $(r=0.41 ; p=0.007)$ in controls but not in patients. There were no significant correlations between P1 and evoked beta and alpha activity. There was no correlation between chlorpromazine equivalents and evoked oscillatory activity during encoding.

\section{Delay}

During the delay period, we found significant changes compared with baseline in induced alpha and gamma band activity. Figure 4 depicts the grand mean induced alpha band at posterior electrodes and induced gamma band activity at anterior electrodes during the delay period of 500-4500 ms after stimulus offset. We divided the delay period into an early delay window between 500 and $2500 \mathrm{~ms}$ and into a late delay window between 2500 and 4500 ms (supplemental Table 2, available at www.jneurosci.org as supplemental material). There were no significant differences in latency in induced alpha and gamma band activity during both delay periods.

\section{Early delay}

The mean induced alpha band amplitude was significantly stronger at posterior electrodes compared with anterior electrodes $\left(F_{(1,26)}=38.64 ; p<0.001\right)$, whereas the mean induced gamma band amplitude was, in trend, stronger at anterior electrodes $\left(F_{(1,26)}\right.$ $=4.18 ; p=0.051)$. Patients and controls did not differ in induced alpha and gamma band activity. However, both groups showed an increase in induced alpha and in induced gamma band activity with increasing WM load (alpha: $F_{(2,25)}=5.55, p=0.008$; gamma: $\left.F_{(2,25)}=3.82, p=0.041\right)$. Post hoc tests confirmed that the increase with WM load was linear in induced gamma band activity (linear increase, WM load: $F_{(1,26)}=4.84 ; p=0.037$ ) for both groups but was only linear in controls for induced alpha band activity (linear increase, WM load $\times$ group: $F_{(2,25)}=4.36 ; p=$ $0.047)$. There was a negative correlation between chlorpromazine equivalents and induced alpha band activity for WM load 3 ( $r=$ $-0.67 ; p=0.0012$ ). 

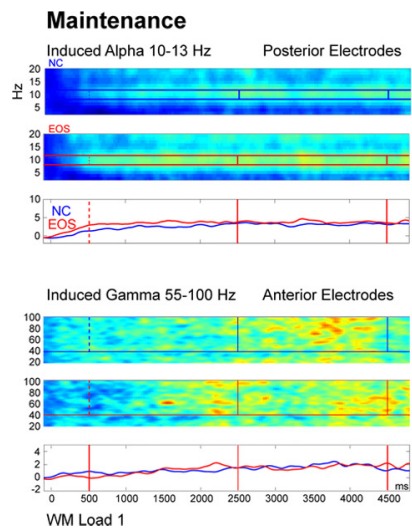
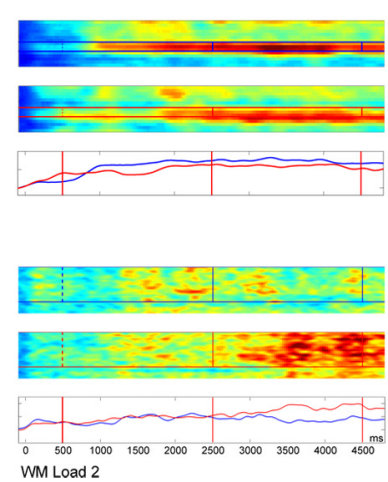
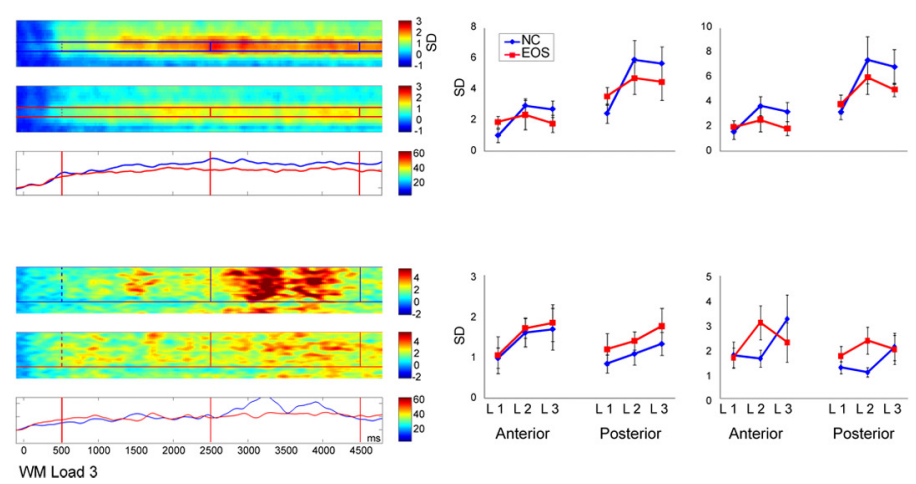

Figure 4. Induced oscillatory activity during WM maintenance. Induced alpha activity (top) at posterior electrodes and gamma activity (bottom) at anterior electrodes during the maintenance following the offset of the first sample stimulus for WM load 1 (left), the offset of the second stimulus for WM load 2 (center), and the offset of the third stimulus for WM load 3 (right) are shown for the early delay (500-2500 ms) and the late delay (2500 - $4500 \mathrm{~ms}$ ) periods for controls (NC, blue, top row) and patients with EOS (red, middle row) and the group comparison in the frequency range of interest (bottom row). On the far right, mean amplitudes in response to WM load 1, 2, or 3 during early and late delay are shown for the anterior and posterior ROI for patients (EOS, red) and controls (NC, blue). Error bars represent SE.

\section{Late delay}

Similar to the early delay, induced alpha band activity was significantly stronger at posterior electrodes $\left(F_{(1,26)}=34.52 ; p<\right.$ $0.001)$, whereas induced gamma band activity was again stronger at anterior electrodes $\left(F_{(1,26)}=7.29 ; p=0.012\right)$. As for the early delay, neither induced alpha nor gamma band activity did differ between groups, but induced alpha band activity increased with increasing WM load $\left(F_{(2,25)}=6.46 ; p=0.004\right)$ at posterior electrodes $\left(\right.$ WM load $\times$ ROI: $F_{(2,25)}=4.55 ; p=0.023$ ) (Fig. 4). In contrast, induced gamma band activity showed an interaction between WM load $\times$ group that was, in trend, significant $\left(F_{(2,25)}=\right.$ $2.97 ; p=0.079)$. Post hoc tests indicated that this may be attributable to a significant quadratic decrease (increase in induced gamma band activity for WM load 3 ) in controls and a quadratic increase (increase for WM load 2 followed by decrease for WM load 3) in patients with increasing WM load (linear contrast, WM load $\times$ group: $\left.F_{(2,25)}=11.44 ; p=0.002\right)$ (Fig. 4$)$. There was no correlation between chlorpromazine equivalents and induced alpha and gamma band activity during late delay.

\section{Retrieval}

In both groups, the probe stimulus elicited evoked theta band activity between 30 and $500 \mathrm{~ms}$ and induced theta and gamma band activity between 200 and $600 \mathrm{~ms}$ and between 200 and 800 ms, respectively (supplemental Table 3, available at www.jneurosci.org as supplemental material). The grand mean evoked and induced theta band activity at anterior electrodes and induced gamma band activity at posterior electrodes in response to WM loads 1-3 during retrieval in controls and patients are illustrated in Figure 5.

\section{Evoked activity}

Evoked theta band activity did not show any difference in latency, and there was no significant amplitude difference between anterior and posterior electrodes. Patients showed significant mean amplitude reductions in evoked theta band activity $\left(F_{(1,26)}=\right.$ $6.63 ; p=0.016)$. There were no further differences between WM load conditions for evoked theta band activity.

\section{Induced activity}

Induced theta band activity peaked earlier at posterior compared with anterior electrodes $\left(F_{(1,26)}=8.15 ; p=0.008\right)$, whereas induced gamma band activity did not show any difference in latency.
The mean induced theta band activity was significantly stronger in amplitude at anterior electrodes $\left(F_{(1,26)}=58.93 ; p<\right.$ $0.001)$, and the mean induced gamma band activity was significantly stronger at posterior electrodes $\left(F_{(1,26)}=16.85 ; p<\right.$ $0.001)$. Both induced theta $\left(F_{(1,26)}=9.28 ; p=0.005\right)$ and gamma $\left(F_{(1,26)}=5.22 ; p=0.03\right)$ band activity levels were reduced in patients compared with controls.

Finally, the mean amplitude of induced theta band activity decreased with the increase in WM load $\left(F_{(1,26)}=5.28 ; p=0.01\right)$ in both groups. This was explained by a quadratic decrease at posterior electrodes $\left(\mathrm{WM}\right.$ load $\times$ ROI: $\left.F_{(2,25)}=9.45 ; p=0.001\right)$. There was no WM load effect on induced gamma band activity.

\section{Correlation between behavioral data and frequency components}

Hierarchical linear regression analyses were computed for frequency bands that either showed a group or a WM load effect to test whether the number of successfully encoded items can be predicted by any of the frequency components during encoding (block 1: evoked posterior beta, evoked posterior alpha, and evoked anterior theta), during delay (block 2: induced early and late delay anterior gamma and induced early and late posterior alpha), and during retrieval (block 3: evoked and induced anterior theta and induced posterior gamma activity). Only activity during encoding but not during delay or the retrieval period predicted the number of successfully memorized items. During encoding, evoked posterior beta, alpha, and anterior theta band activity $\left(F_{(2,25)}=6.91 ; p=0.001 ; R^{2}: 0.35\right)$ predicted the number of successfully encoded items in controls but not in patients. Whereas the number of encoded items was negatively correlated with evoked beta and theta band activity (standardized beta: $-0.590, p<0.001$; and $-0.301, p=0.031$, respectively), it was positively correlated with evoked alpha band activity (standardized beta: $0.449 ; p=0.005$ ).

\section{Discussion}

This study investigated evoked and induced oscillatory EEG activity in a broad frequency range in patients with EOS and healthy controls during the three phases of visual WM.

Compared with controls, patients showed changes in oscillatory activity in all three phases of a visual WM task. In controls, evoked activity in theta, alpha, and beta band activity during 


\section{Retrieval}
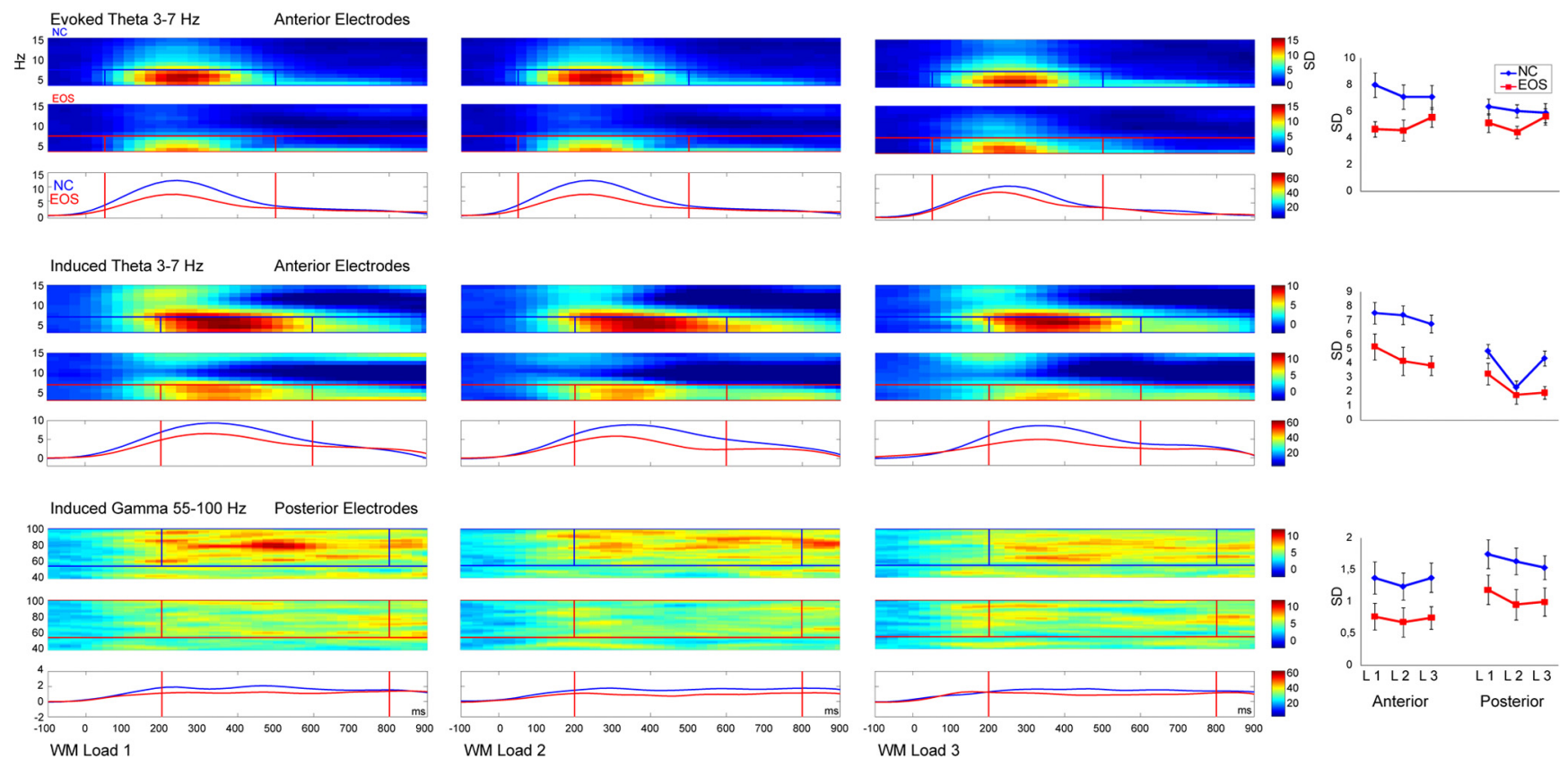

Figure 5. Induced oscillatory activity during WM retrieval. Evoked (top) and induced (middle) theta activity at anterior electrodes and induced gamma activity (bottom) at posterior electrodes following the test stimulus for WM load 1 (left), WM load 2 (center), and WM load 3 (right) are shown for the retrieval period of 1 s for controls (NC, blue, top row) and patients with EOS (red, middle row) and the group comparison in the frequency range and time window of interest (bottom row). The frequency and latency range of interest are denoted in bars. On the far right, mean amplitudes in response to WM load 1, 2, or 3 are shown for the anterior and posterior ROI for patients (EOS, red) and controls (NC, blue). Error bars represent SE.

encoding predicted the number of successfully encoded items. Patients showed reduced evoked activity in these frequency bands. During the late maintenance period, patients showed an increase in induced gamma band amplitude in response to WM load 2 and failed to sustain induced gamma band activity for the highest WM load. In contrast, controls showed an increase in induced band gamma amplitude in response to WM load 3. This is consistent with patients experiencing difficulties in WM tasks already at lower memory loads.

\section{Encoding}

During encoding, patients showed reduced evoked oscillatory activity in a frequency range between 3 and $30 \mathrm{~Hz}$ (theta, alpha, and beta band activity) compared with controls.

Using hierarchical regression including encoding, maintenance, and retrieval in separate blocks, we found that only WM encoding activity significantly predicted performance. Such a relationship was only found for the control group.

Impairments in evoked oscillatory activity may be explained by a reduced precision in the transmission of transient signals, and thus by a reduced signal-to-noise ratio. Alternatively, a reduction in stimulus-induced phase resetting of ongoing rhythmic activity in each trial may lead to impairments in evoked oscillatory and the P1/N1 complex (Makeig et al., 2002; Klimesch et al., 2007). Thus, the deficits in evoked oscillatory activity in the patients may be related to the recent finding of a P1 deficit during WM encoding (Haenschel et al., 2007b). This interpretation is supported by the present finding of a relationship between posterior theta activity and the P1 component in controls but not in patients.

Controls showed a decrease in evoked theta and beta activity with increasing WM load, whereas patients showed a general reduction compared with controls and no changes with WM load.
The reduction of evoked beta band activity with increasing WM load in controls could be caused by a strong response to the first stimulus followed by habituation in response to the second and the third stimulus (Haenschel et al., 2000). In that case, the reduced evoked beta band activity in patients may be attributable to an inability to entrain the neural system to the first item in a sequence of stimuli. A similar inability to respond to the first stimulus with an increase in evoked theta and beta band activity and to habituate to the second stimulus has been found in auditory sensory gating studies (Hong et al., 2004a; Jansen et al., 2004; Brockhaus-Dumke et al., 2008).

It is of interest to note that there is increasing evidence for some degree of independence of the local circuits and mechanisms underlying beta $1(12-20 \mathrm{~Hz})$ and beta $2(20-30 \mathrm{~Hz})$ (Roopun et al., 2008), which may also be reflected in some differences in their cognitive functions (Uhlhaas et al., 2008). However, post hoc analysis separating the power changes in the beta 1 and beta 2 subbands found no significant modulation across conditions, which suggests that the effects reported above were more widely distributed across the beta band range.

\section{Early delay}

Contrary to the proposal of Lee and Park (2005), we did not observe any group difference in the early delay activity. The increase in induced alpha and gamma band activity during the delay period in association with WM load complements previous findings of WM maintenance-related oscillatory activity (TallonBaudry et al., 1998; Jensen et al., 2002; Busch and Herrmann, 2003; Howard et al., 2003; Leiberg et al., 2006). In addition, increased induced gamma band activity has been described during the short-term consolidation of letters into WM that lasted for several hundred milliseconds (Mainy et al., 2007) and during maintenance reflecting the sustained activity necessary for stimulus feature representations (Kaiser et al., 2008). This may indi- 
cate that both groups used the same resources for the short-term consolidation and early maintenance of the stimulus representations in WM. Interestingly, Fuller et al. $(2005,2009)$ provided behavioral evidence that the consolidation of perceptual representations in WM is slowed in patients with schizophrenia. Our results would suggest that this is primarily attributable to impairments in evoked oscillations during encoding resulting in less salient (i.e., noisier) perceptual representations that impair WM consolidation.

\section{Late delay}

Most of the previous EEG studies of WM used a much shorter delay between the sample and probe stimuli and were thus not able to capture any differential processes during prolonged delay periods [see Meltzer et al. (2008) for a 6 s delay window]. Induced gamma band activity in the later delay window showed a trend toward divergent patterns of activity between the groups, with an increase in induced gamma band activity from WM load 2 to load 3 in controls and from WM load 1 to load 2 in patients and a decrease from WM load 2 to load 3 in patients. Interestingly, Basar-Eroglu et al. (2007) also reported hyperactivation of evoked gamma band activity for lower WM task demands in patients in a combined cognitive control and N-back task. The inverted U-shaped function of load-dependent gamma activity of the patients also bears intriguing resemblance to similar fMRI findings (Callicott et al., 2003; Karlsgodt et al., 2009), which indicate that the cortical storage system reaches its limit at lower WM demands. This interpretation is supported by the interaction between WM load and group in our behavioral data. However, because of the poor spatial resolution of EEG, this pattern could be explained by the mean of all sources contributing to the induced gamma band activity.

The inverted U-shaped pattern only emerged during the latter part of the delay, which is consistent with the observation by Reilly et al. (2006) that spatial WM deficits of patients became apparent only at longer delay periods (from $3 \mathrm{~s}$ to the maximum impairments at $8 \mathrm{~s}$ ). Additional studies with such long delay periods are needed for an understanding of the mechanisms of WM rehearsal and decay.

\section{Retrieval}

Compared with controls, patients showed reduced evoked and induced theta and induced gamma band activity during retrieval. Given that induced theta band activity is larger for old compared with new words during recognition (Klimesch et al., 1997), this could be an indication that controls recognized the previously stored items, whereas patients might have treated them as novel. Induced gamma band activity has been suggested to be a signature of cortical object representation, and an increase in induced gamma band activity over posterior electrodes has been linked to successful recognition of complex meaningful objects (Gruber and Muller, 2005, 2006; Lachaux et al., 2005). The reduction of induced gamma band activity thus suggests that, during retrieval, patients were less able to retrieve the representation from WM. However, our data did not show a relationship between the amount of induced gamma activity during retrieval and successful recognition: whereas the group difference in induced gamma band activity was already evident in patients for WM load 1, behaviorally patients were equally successful for this WM load.

A limitation of this study is that all the patients were receiving medication at the time of testing. However, the fact that we found no correlation with individual chlorpromazine equivalents in line with previous studies on oscillatory activity (Gallinat et al., 2004; Hong et al., 2004b) in schizophrenia suggests that the impairments in oscillatory activity in our sample of patients with EOS are unlikely to reflect the effects of medication.

Furthermore, because we studied a group of adolescent patients with a relatively recent onset of illness, factors such as long illness duration, chronic medication, and higher sample heterogeneity should have confounded our study to a lesser degree than the majority of studies investigating adult patients with chronic schizophrenia.

We would thus suggest that changes in oscillatory EEG activity reflect some of the neurodevelopmental processes leading to schizophrenia. Such a neurodevelopmental account has also been suggested for the putative underlying neurochemical and local circuit changes (Lewis and González-Burgos, 2008; Uhlhaas et al., 2009). However, we do not exclude that the progression of the disease is characterized by additional neural changes that lead to further impairments of neural synchronization, which has indeed been suggested by a recent study that compared induced highfrequency oscillatory activity during the perception of Mooney faces in controls and first-episode and chronic patients. This study reported a progressive deficit in oscillatory activity during the course of the disorder (Tillmann et al., 2008).

\section{General conclusions and implications}

The design of our study, which included the parametric manipulation of WM load and a long delay interval, allowed us to extend previous work on the relationship between oscillatory activity and WM impairments in schizophrenia in several ways. First, our results indicate impairments in all WM phases in a broad frequency range, whereas previous studies mostly focused on WM maintenance. This highlights the importance of investigating the contribution of the different WM phases to performance.

Second, the analysis of a $5 \mathrm{~s}$ delay period suggests that impairments in oscillatory activity may become more evident during longer maintenance intervals. One possibility is that, during longer maintenance, additional processes related to active rehearsal may become important, which may be more susceptible to disturbance in patients with schizophrenia. Finally, the missing relationship between evoked activity and performance in patients indicates the functional significance of early evoked oscillatory impairments during encoding for the understanding of the disorder.

This raises the question whether evoked oscillatory activity may, in the future, be used to assess treatment response and may serve as a tool in early diagnosis. Furthermore, our results have potentially important implications for cognitive rehabilitation programs. Cognitive remediation therapy has been associated with moderate improvements in WM (Wykes et al., 2007a,b) as measured with the Digit Span. Our present and previous (Uhlhaas et al., 2006; Haenschel et al., 2007b) data suggest that additional visual perceptual training may enhance these effects.

Our results also have implications for the pathophysiology of schizophrenia. Lewis (2000) suggested that altered GABA neurotransmission in the DLPFC may lead to impaired oscillatory activity and as a consequence to WM deficits. Hashimoto et al. (2008) have now extended the finding of altered GABA neurotransmission to multiple cortical regions including primary visual cortices. Indeed, by now there is evidence that alteration in markers of the GABA system and deficits in NMDA receptormediated excitation of these interneurons result in pathorhythmogenesis within specific frequency bands using animal models of schizophrenia (Cunningham et al., 2006; Roopun et al., 2008). Our finding of widespread reduced evoked oscillatory 
activity during WM encoding may thus relate to such altered GABAergic neurotransmission and abnormal NMDA receptormediated glutamatergic inputs onto these GABA neurons (Lewis and Moghaddam, 2006). Oscillatory EEG (and magnetoencephalography) activity is probably the only noninvasive neurophysiological measure that allows for direct comparison with neuronal firing models (Whittington et al., 1997; Doheny et al., 2000; Haenschel et al., 2000) and is thus uniquely suited for the translational testing of molecular and cellular models of schizophrenia.

\section{References}

Bachman P, Kim J, Yee C, Therman S, Manninen M, Lönnqvist J, Kaprio J, Huttunen M, Näätänen R, Cannon T (2008) Abnormally high EEG alpha synchrony during working memory maintenance in twins discordant for schizophrenia. Schizophr Res 103:293-297.

Badcock JC, Badcock DR, Read C, Jablensky A (2008) Examining encoding imprecision in spatial working memory in schizophrenia. Schizophr Res 100:144-152.

Basar-Eroglu C, Brand A, Hildebrandt H, Karolina Kedzior K, Mathes B, Schmiedt C (2007) Working memory related gamma oscillations in schizophrenia patients. Int J Psychophysiol 64:39-45.

Brockhaus-Dumke A, Mueller R, Faigle U, Klosterkoetter J (2008) Sensory gating revisited: relation between brain oscillations and auditory evoked potentials in schizophrenia. Schizophr Res 99:238-249.

Busch NA, Herrmann CS (2003) Object-load and feature-load modulate EEG in a short-term memory task. Neuroreport 14:1721-1724.

Callicott J, Mattay V, Verchinski B, Marenco S, Egan M, Weinberger D (2003) Complexity of prefrontal cortical dysfunction in schizophrenia: more than up or down. Am J Psychiatry 160:2209-2215.

Cannon T, Glahn D, Kim J, Van Erp T, Karlsgodt K, Cohen M, Nuechterlein K, Bava S, Shirinyan D (2005) Dorsolateral prefrontal cortex activity during maintenance and manipulation of information in working memory in patients with schizophrenia. Arch Gen Psychiatry 62:1071-1080.

Cho RY, Konecky RO, Carter CS (2006) Impairments in frontal cortical gamma synchrony and cognitive control in schizophrenia. Proc Natl Acad Sci U S A 103:19878-19883.

Cowan N, Elliott E, Scott Saults J, Morey C, Mattox S, Hismjatullina A, Conway A (2005) On the capacity of attention: its estimation and its role in working memory and cognitive aptitudes. Cogn Psychol 51:42-100.

Cunningham M, Hunt J, Middleton S, LeBeau F, Gillies M, Gillies M, Davies C, Maycox P, Whittington M, Racca C (2006) Region-specific reduction in entorhinal gamma oscillations and parvalbumin-immunoreactive neurons in animal models of psychiatric illness. J Neurosci 26:2767-2776.

Doheny HC, Faulkner HJ, Gruzelier JH, Baldeweg T, Whittington MA (2000) Pathway-specific habituation of induced gamma oscillations in the hippocampal slice. Neuroreport 11:2629-2633.

Fuller RL, Luck SJ, McMahon RP, Gold JM (2005) Working memory consolidation is abnormally slow in schizophrenia. J Abnorm Psychol 114:279-290.

Fuller RL, Luck SJ, Braun EL, Robinson BM, McMahon RP, Gold JM (2009) Impaired visual working memory consolidation in schizophrenia. Neuropsychology 23:71-80.

Gallinat J, Winterer G, Herrmann CS, Senkowski D (2004) Reduced oscillatory gamma-band responses in unmedicated schizophrenic patients indicate impaired frontal network processing. Clin Neurophysiol 115:1863-1874.

Goldman-Rakic P (2001) Working memory dysfunction in schizophrenia. In: The frontal lobes and neuropsychiatric illness (Salloway SP, Malloy PF, Duffy JD, eds), pp 71-82. Washington, DC: American Psychiatric.

Gooding DC, Tallent KA (2004) Nonverbal working memory deficits in schizophrenia patients: evidence of a supramodal executive processing deficit. Schizophr Res 68:189-201.

Gruber T, Muller MM (2005) Oscillatory brain activity dissociates between associative stimulus content in a repetition priming task in the human EEG. Cereb Cortex 15:109-116.

Gruber T, Muller MM (2006) Oscillatory brain activity in the human EEG during indirect and direct memory tasks. Brain Res 1097:194-204.

Gruber WR, Klimesch W, Sauseng P, Doppelmayr M (2005) Alpha phase synchronization predicts P1 and N1 latency and amplitude size. Cereb Cortex 15:371-377.
Haenschel C, Baldeweg T, Croft RJ, Whittington M, Gruzelier J (2000) Gamma and beta frequency oscillations in response to novel auditory stimuli: a comparison of human electroencephalogram (EEG) data with in vitro models. Proc Natl Acad Sci U S A 97:7645-7650.

Haenschel C, Uhlhaas P, Singer W (2007a) Synchronous oscillatory activity and working memory in schizophrenia. Pharmacopsychiatry 40:54-61.

Haenschel C, Bittner RA, Haertling F, Rotarska-Jagiela A, Maurer K, Singer W, Linden DE (2007b) Contribution of impaired early-stage visual processing to working memory dysfunction in adolescents with schizophrenia: a study with event-related potentials and functional magnetic resonance imaging. Arch Gen Psychiatry 64:1229-1240.

Hashimoto T, Bazmi HH, Mirnics K, Wu Q, Sampson AR, Lewis DA (2008) Conserved regional patterns of GABA-related transcript expression in the neocortex of subjects with schizophrenia. Am J Psychiatry 165:479-489.

Hong LE, Summerfelt A, McMahon RP, Thaker GK, Buchanan RW (2004a) Gamma/beta oscillation and sensory gating deficit in schizophrenia. Neuroreport 15:155-159.

Hong LE, Summerfelt A, McMahon R, Adami H, Francis G, Elliott A, Buchanan RW, Thaker GK (2004b) Evoked gamma band synchronization and the liability for schizophrenia. Schizophr Res 70:293-302.

Howard MW, Rizzuto DS, Caplan JB, Madsen JR, Lisman J, AschenbrennerScheibe R, Schulze-Bonhage A, Kahana MJ (2003) Gamma oscillations correlate with working memory load in humans. Cereb Cortex 13:1369-1374.

Jansen BH, Hegde A, Boutros NN (2004) Contribution of different EEG frequencies to auditory evoked potential abnormalities in schizophrenia. Clin Neurophysiol 115:523-533.

Javitt DC, Strous RD, Grochowski S, Ritter W, Cowan N (1997) Impaired precision, but normal retention, of auditory sensory ("echoic") memory information in schizophrenia. J Abnorm Psychol 106:315-324.

Jensen O, Gelfand J, Kounios J, Lisman JE (2002) Oscillations in the alpha band $(9-12 \mathrm{~Hz})$ increase with memory load during retention in a shortterm memory task. Cereb Cortex 12:877-882.

Kaiser J, Heidegger T, Wibral M, Altmann CF, Lutzenberger W (2008) Distinct gamma-band components reflect the short-term memory maintenance of different sound lateralization angles. Cereb Cortex 18:2286-2295.

Karlsgodt K, Sanz J, van Erp T, Bearden C, Nuechterlein K, Cannon T (2009) Re-evaluating dorsolateral prefrontal cortex activation during working memory in schizophrenia. Schizophr Res 108:143-150.

Kay S, Fiszbein A, Opler L (1987) The positive and negative syndrome scale (PANSS) for schizophrenia. Schizophr Bull 13:261-276.

Klimesch W, Doppelmayr M, Schimke H, Ripper B (1997) Theta synchronization and alpha desynchronization in a memory task. Psychophysiology 34:169-176.

Klimesch W, Schack B, Schabus M, Doppelmayr M, Gruber W, Sauseng P (2004) Phase-locked alpha and theta oscillations generate the P1-N1 complex and are related to memory performance. Brain Res Cogn Brain Res 19:302-316.

Klimesch W, Sauseng P, Hanslmayr S (2007) EEG alpha oscillations: the inhibition-timing hypothesis. Brain Res Rev 53:63-88.

Kwon JS, O’Donnell BF, Wallenstein GV, Greene RW, Hirayasu Y, Nestor PG, Hasselmo ME, Potts GF, Shenton ME, McCarley RW (1999) Gamma frequency-range abnormalities to auditory stimulation in schizophrenia. Arch Gen Psychiatry 56:1001-1005.

Lachaux JP, Rodriguez E, Martinerie J, Varela FJ (1999) Measuring phase synchrony in brain signals. Hum Brain Mapp 8:194-208.

Lachaux JP, George N, Tallon-Baudry C, Martinerie J, Hugueville L, Minotti L, Kahane P, Renault B (2005) The many faces of the gamma band response to complex visual stimuli. Neuroimage 25:491-501.

Lee J, Park S (2005) Working memory impairments in schizophrenia: a meta-analysis. J Abnorm Psychol 114:599-611.

Lee J, Folley B, Gore J, Park S (2008) Origins of spatial working memory deficits in schizophrenia: an event-related FMRI and near-infrared spectroscopy study. PLoS ONE 3:e1760.

Lehrl S (1995) Mehrfachwahl-Wortschatz-Intelligenztest. Erlangen, Germany: Straube.

Leiberg S, Lutzenberger W, Kaiser J (2006) Effects of memory load on cortical oscillatory activity during auditory pattern working memory. Brain Res 1120:131-140.

Lencz T, Bilder RM, Turkel E, Goldman RS, Robinson D, Kane JM, Lieberman JA (2003) Impairments in perceptual competency and maintenance on a visual 
delayed match-to-sample test in first-episode schizophrenia. Arch Gen Psychiatry 60:238-243.

Lewis D, González-Burgos G (2008) Neuroplasticity of neocortical circuits in schizophrenia. Neuropsychopharmacology 33:141-165.

Lewis D, Moghaddam B (2006) Cognitive dysfunction in schizophrenia: convergence of gamma-aminobutyric acid and glutamate alterations. Arch Neurol 63:1372-1376.

Lewis DA (2000) GABAergic local circuit neurons and prefrontal cortical dysfunction in schizophrenia. Brain Res Brain Res Rev 31:270-276.

Light G, Hsu J, Hsieh M, Meyer-Gomes K, Sprock J, Swerdlow N, Braff D (2006) Gamma band oscillations reveal neural network cortical coherence dysfunction in schizophrenia patients. Biol Psychiatry 60:1231-1240.

Linden DE, Bittner RA, Muckli L, Waltz JA, Kriegeskorte N, Goebel R, Singer W, Munk MH (2003) Cortical capacity constraints for visual working memory: dissociation of fMRI load effects in a fronto-parietal network. Neuroimage 20:1518-1530.

Mainy N, Kahane P, Minotti L, Hoffmann D, Bertrand O, Lachaux JP (2007) Neural correlates of consolidation in working memory. Hum Brain Mapp 28:183-193.

Makeig S, Westerfield M, Jung TP, Enghoff S, Townsend J, Courchesne E, Sejnowski TJ (2002) Dynamic brain sources of visual evoked responses. Science 295:690-694.

Melloni L, Schwiedrzik C, Wibral M, Rodriguez E, Singer W (2009) Response to: Yuval-Greenberg et al., "Transient induced gamma-band response in EEG as a manifestation of miniature saccades." Neuron 58, 429-441. Neuron 62:8-10; author reply 10-12.

Meltzer J, Zaveri H, Goncharova I, Distasio M, Papademetris X, Spencer S, Spencer D, Constable R (2008) Effects of working memory load on oscillatory power in human intracranial EEG. Cereb Cortex 18:1843-1855.

Pashler H (1988) Familiarity and visual change detection. Percept Psychophys 44:369-378.

Reilly J, Harris M, Keshavan M, Sweeney J (2006) Adverse effects of risperidone on spatial working memory in first-episode schizophrenia. Arch Gen Psychiatry 63:1189-1197.

RodriguezE, George N, Lachaux JP, Martinerie J, Renault B, Varela FJ (1999) Perception's shadow: long-distance synchronization of human brain activity. Nature 397:430-433.

Roopun A, Cunningham M, Racca C, Alter K, Traub R, Whittington M (2008) Region-specific changes in gamma and beta2 rhythms in NMDA receptor dysfunction models of schizophrenia. Schizophr Bull 34:962-973.

Schmiedt C, Brand A, Hildebrandt H, Basar-Eroglu C (2005) Event-related theta oscillations during working memory tasks in patients with schizophrenia and healthy controls. Brain Res Cogn Brain Res 25:936-947.
Silver H, Feldman P, Bilker W, Gur RC (2003) Working memory deficit as a core neuropsychological dysfunction in schizophrenia. Am J Psychiatry 160:1809-1816.

Singer W (1999) Neuronal synchrony: a versatile code for the definition of relations? Neuron 24:49-65, 111-125.

Spencer KM, Nestor PG, Niznikiewicz MA, Salisbury DF, Shenton ME, McCarley RW (2003) Abnormal neural synchrony in schizophrenia. J Neurosci 23:7407-7411.

Tallon-Baudry C, Bertrand O, Peronnet F, Pernier J (1998) Induced gamma-band activity during the delay of a visual short-term memory task in humans. J Neurosci 18:4244-4254.

Tek C, Gold J, Blaxton T, Wilk C, McMahon RP, Buchanan RW (2002) Visual perceptual and working memory impairments in schizophrenia. Arch Gen Psychiatry 59:146-153.

Tillmann C, Wibral M, Leweke M, Kohler A, Singer W, Koethe D, Kranaster L, Maurer K, Uhlhaas P (2008) Source localization of high-frequency oscillations reveals widespread reductions in gamma-band activity during perceptual organisation in chronic and first-episode schizophrenia. Soc Neurosci Abstr 34:54.2.

Uhlhaas P, Roux F, Singer W, Haenschel C, Sireteanu R, Rodriguez R (2009) The development of neural synchrony reflects late maturation and restructuring of functional networks in humans. Proc Natl Acad Sci U S A 106:9866-9871.

Uhlhaas PJ, Linden DE, Singer W, Haenschel C, Lindner M, Maurer K, Rodriguez E (2006) Dysfunctional long-range coordination of neural activity during Gestalt perception in schizophrenia. J Neurosci 26:8168-8175.

Whittington M, Traub R, Faulkner H, Stanford I, Jefferys J (1997) Recurrent excitatory postsynaptic potentials induced by synchronized fast cortical oscillations. Proc Natl Acad Sci U S A 94:12198-12203.

Whittington MA, Traub RD, Jefferys JG (1995) Synchronized oscillations in interneuron networks driven by metabotropic glutamate receptor activation. Nature 373:612-615.

Woods SW (2003) Chlorpromazine equivalent doses for the newer atypical antipsychotics. J Clin Psychiatry 64:663-667.

Wykes T, Newton E, Landau S, Rice C, Thompson N, Frangou S (2007a) Cognitive remediation therapy (CRT) for young early onset patients with schizophrenia: an exploratory randomized controlled trial. Schizophr Res 94:221-230.

Wykes T, Reeder C, Landau S, Everitt B, Knapp M, Patel A, Romeo R (2007b) Cognitive remediation therapy in schizophrenia: randomised controlled trial. Br J Psychiatry 190:421-427.

Yuval-Greenberg S, Tomer O, Keren A, Nelken I, Deouell L (2008) Transient induced gamma-band response in EEG as a manifestation of miniature saccades. Neuron 58:429-441. 